\title{
Analysis of Chlorhexidine Modified Cement in Orthodontic Patients: A Double-Blinded, Randomized, Controlled Trial
}

\author{
José Lucas dos Santos Araújo ${ }^{1,2}$ Mariana Massi Afonso Alvim²,3 Márcio José da Silva Campos 2,4 \\ Ana Carolina Morais Apolônio ${ }^{2,4}$ Fabíola Galbiatti Carvalho ${ }^{2,4}$ Rogério Lacerda-Santos $2,4, \odot$
}

${ }^{1}$ Graduate Program in Dentistry, Dental School, Federal University

Address for correspondence Rogério Lacerda-Santos, DDS, MSD, of Juiz de Fora, Juiz de Fora, Minas Gerais, Brazil

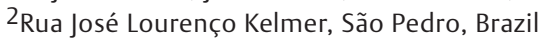

${ }^{3}$ Pharmacy School, Federal University of Juiz de Fora, Juiz de Fora, Minas Gerais, Brazil

${ }^{4}$ Department of Orthodontics, Faculty of Dentistry, Federal

University of Juiz de Fora, Minas Gerais, Brazil \begin{abstract}
PhD, Department of Orthodontics, Faculty of Dentistry, Federal University of Juiz de Fora, Avenue Doutor Raimundo Monteiro Rezende, n.330, Centro, Governador Valadares, MG 35010-177, Brazil (e-mail: lacerdaorto@hotmail.com; lacerdaorto@gmail.com).
\end{abstract}

Eur J Dent 2021;15:639-646.

\begin{abstract}
Keywords

- glass ionomer cement

- chlorhexidine

- microbiota

- orthodontic

Objectives The aim of this study was to evaluate the microbiological and mechanical properties of glass ionomer cement (GIC) modified by chlorhexidine (CLX) for the purpose of cementing bands to the teeth of orthodontic patients.

Materials and Methods Ten patients, between the ages of 19 and 33 years, in the initial stage of orthodontic treatment, were randomly designated to two groups using the split-mouth design $(n=10)$. One group (GICEX) had bands cemented with GIC modified by CLX and a Control group (GIC), evaluated at time intervals before (T0), 3 months (T3), and 6 months (T6) after cementation. Total microbiological counts were performed, and color stability of tooth enamel, salivary $\mathrm{pH}$, and the adhesive remnant index (ARI) were evaluated.

Statistical Analysis The Friedman and Dunn's tests, Mann-Whitney, one-way analysis of variance, and Tukey, and paired and non-paired $t$-tests $(p<0.05)$ were used.

Results In T3, there was evidence of significant reduction in the quantity of colony forming unit (CFU) in GICEX group in comparison with the Control $(p=0.041)$. In T6, the quantity of CFU was similar to the quantity in T3 and significantly different to control $(p=0.045)$; Control group demonstrated a similar quantity of CFU between the experimental time intervals $(p=0.066)$. Salivary $\mathrm{pH}$ demonstrated significant difference only between the time intervals T0 and T6 $(p=0.022)$. The tooth enamel color $(p=0.366)$ and ARI $(p=0.343)$ values demonstrated no significant changes.

Conclusion The incorporation of CLX into GIC demonstrated effective antibacterial action, allowed a good bond of the cement to the enamel, a high rate of survival of the bands, did not change the color of the tooth enamel, and maintained the salivary $\mathrm{pH}$ at physiological levels.
\end{abstract}

published online August 24, 2021
DOI https://doi.org/

$10.1055 / \mathrm{s}-0041-1727556$ ISSN 1305-7456 (c) 2021. European Journal of Dentistry.

This is an open access article published by Thieme under the terms of the Creative Commons Attribution-NonDerivative-NonCommercial-License, permitting copying and reproduction so long as the original work is given appropriate credit. Contents may not be used for commercial purposes, or adapted, remixed, transformed or built upon. (https://creativecommons.org/licenses/by-nc-nd/4.0/)

Thieme Medical and Scientific Publishers Pvt. Ltd., A-12, 2nd Floor, Sector 2, Noida-201301 UP, India 


\section{Introduction}

Orthodontic banding may lead to microbiological oral changes, due to the formation and accumulation of biofilm around the accessories, ${ }^{1-3}$ triggering caries, gingival inflammations, and risk for patients predisposed to bacterial endocarditis. ${ }^{4}$ Furthermore, deficient oral hygiene favors the proliferation of microorganisms, due to the increase and change in quantity and flow of salivary components, $\mathrm{pH}$ and buffer capacity.,

Dental biofilm may present more than 1,000 bacterial species $^{5}$; however, among the main bacteria present after the placement of orthodontic bands, the most relevant are Streptococcus mutans, Prevotella intermedia and Prevotella loescheii, Capnocytophaga spp., Fusobacterium nucleatum, and Porphyromonas gingivalis. ${ }^{7}$

Among the main materials for cementing orthodontic bands, glass ionomer cement (GIC) is outstanding because it has the characteristics of biocompatibility, bonding to enamel, and absorption and release of fluoride. However, GICs have little action against microorganisms, ${ }^{8,9}$ action that would be beneficial when there is local biofilm accumulation. Different chemical antimicrobial agents have been evaluated with the aim of reducing the progression of dental biofilm, then chlorhexidine digluconate (CLX) has been demonstrated to be effective, has substantivity, and is safe for clinical use in dentistry. ${ }^{1,10}$

CLX is a cationic antiseptic, generally used for mouth washes in the concentration of $0.12 \%$. It belongs to the bisbiguanide group of chemicals (1,1'-hexamethylenebis [5-(4-chlorophenyl)biguanide]). It has a broad spectrum of action against gram positive and negative bacteria and fungi. ${ }^{10}$ It acts by rupturing the cytoplasmic membrane, triggering the loss of vital cell components such as nucleic acid and potassium. Due to the substantivity of CLX on dental biofilm, a reduction in the proliferations of microorganisms ${ }^{11,12}$ occurs due to its bactericidal and bacteriostatic action, impeding the progression of periodontal and caries diseases..$^{10,13}$

The purpose of adding CLX to GIC is to improve the antibacterial action of this material; however, in order for it to be considered adequate for clinical use, it is necessary for it to be biocompatible ${ }^{9,14-16}$ with the tissues. ${ }^{9}$ In vitro studies have demonstrated that the concentration of $10 \%$ CLX is efficacious against $S$. mutans, although at $18 \%$ the antibacterial effect increased without significantly influencing the mechanical properties of diametral tensile strength, resistance to compression, shear bond strength, and microhardness. ${ }^{1,9}$

Although there are in vitro studies about GIC modified by CLX, none of them has evaluated the effect of this modification in orthodontic patients. Therefore, the aim of this study was to evaluate the antimicrobiological and mechanical properties of GIC modified by CLX for the purpose of cementing bands to the teeth of orthodontic patients.

\section{Materials and Methods}

Trial Design, Participants, and Eligibility Criteria

This study was conducted as a prospective, randomized, controlled, double-blind clinical trial, by using a split-mouth design and by treatment with a proportion of allocation of participants of $1: 1$. No change was made in the study design after the study began. This study was conducted in accordance with the guidelines of the Consolidated Standards of Reporting Trials guidelines (-Fig. 1). The project was reviewed and approved by the Ethics Committee on Research with Human Beings of the Federal University of Juiz de Fora, under CAAE number: 08637119.0.0000.5147. For this study, the terms of free and informed consent were obtained from the participants. At the beginning of the study, all the patients were asked to sign the term of consent and the chart containing information about the patient, in complete compliance with the Declaration of Helsinki.

The sample size was defined, based on preliminary pilot study data of five individuals (test: $4.54 \pm 0.25$ colony forming unit [CFU] vs. control: $4.80 \pm 0.10 \mathrm{CFU}$ ) using BioEstat 5.3 Software (Instituto Mamirauá, Belém, Pará, Brazil) and using $\alpha=0.05$ and power of $95 \%$ for a bilateral test, and a minimum sample of six patients was required. Considering the possibility of using nonparametric statistics, $15 \%$ was added, which raised the minimum number of individuals to $7 .{ }^{17}$ A sample loss of $~ 30 \%$ was considered (patients dropping out at some of the time intervals of evaluation), and 3 patients were added, totaling 10 individuals with orthodontic treatment needs. Among the individuals recruited, five were male and five were female, with a mean age of 24.7 years, ranging from 19 to 33 years.

The patients were selected in accordance with the inclusion criteria: not having undergone previous orthodontic treatment, not having used antibiotics in the last 3 months, not making use of systemic medications, not having any systemic disorder that could interfere in the periodontal condition, not having any motor limitations for performing oral hygiene, and not having severe crowding, overjet, and overbite, according to the index of complexity, outcome, and need criteria. ${ }^{18}$ The exclusion criteria were: patients who had periodontal disease and/or caries lesions, clinically and radiographically (RX bite wing) evaluated in any of their teeth.

Randomization was performed by a researcher who did not participate in the clinical part of the study, thereby guaranteeing secrecy of the allocation. The BioEstat 5.3 Software (Instituto Mamirauá) was used to construct a table of randomized numbers, taking into consideration the sample size required in this study, based on a sample of 25 individuals at the beginning of orthodontic treatment. The choice of control and experimental sides was defined by draw.

The patients received basic oral hygiene instructions about the modified Bass technique with the intention of standardizing tooth brushing during the study. They received an oral hygiene kit containing a brush and dental floss (Oral B, São Paulo, Brazil), toothpaste with 1,500 ppm fluoride (Colgate-Palmolive, São Paulo, Brazil), and were instructed not to use mouth washes during the period of evaluation.

In the research, a split-mouth system was adopted, totaling a sample of 20 orthodontic bands (Dental Morelli, Sorocaba, Brazil) of the universal type for mandibular molars.

Blinding of the operator was not possible. Patients were, however, blinded to the group of cement used. Moreover, the 


\section{CONSORT 2010 Flow Diagram}

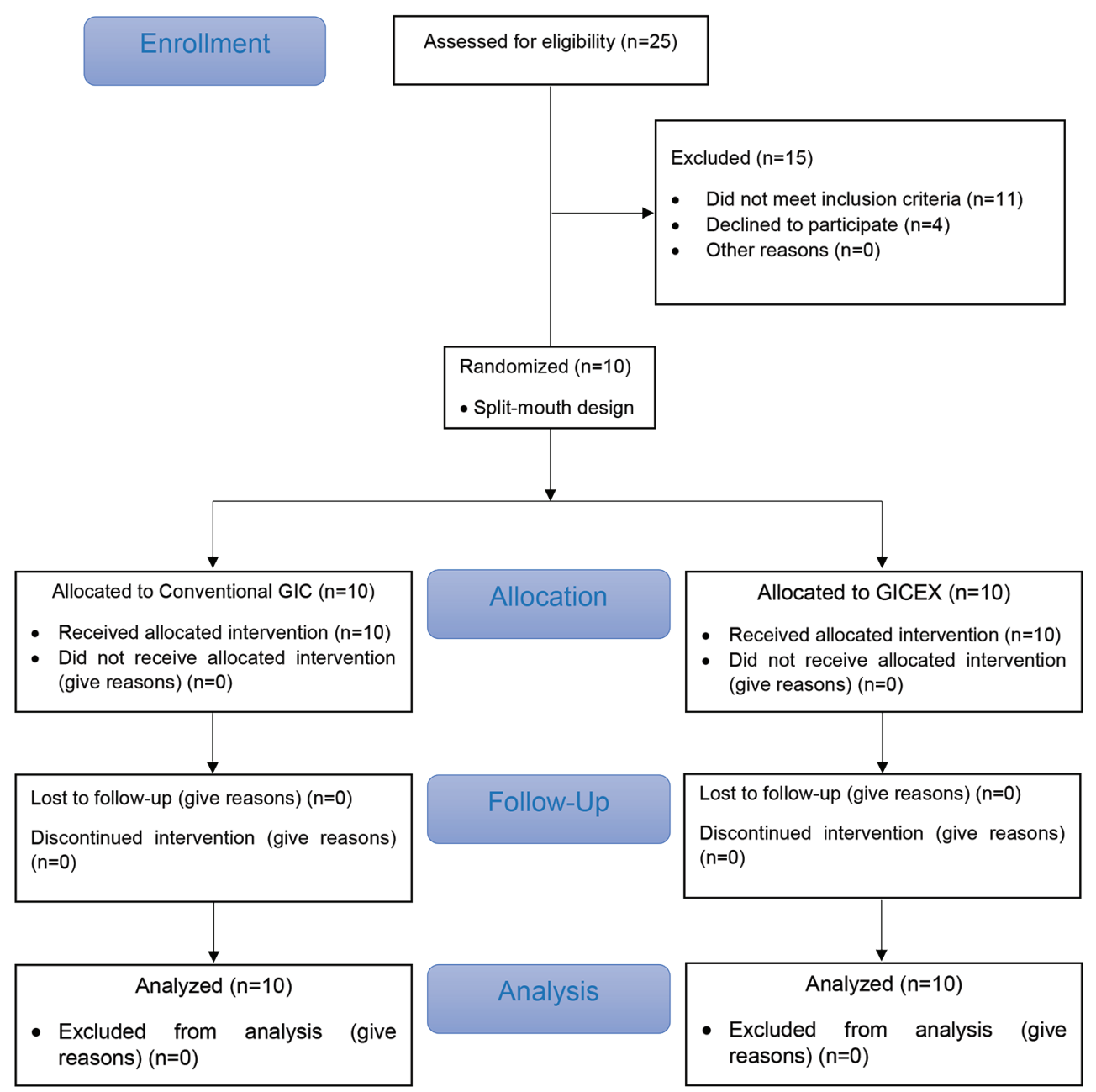

Fig. 1 Flow diagram of the participating patients. GIC, glass ionomer cement.

patients received randomized numbers as identification, and the evaluator of the outcome was incapable of identifying the group to which the individuals belonged, thus being blind to the allocation of treatment. Therefore, patients and data were blindly evaluated, supporting the double-blind design of the study.

\section{Glass Ionomer Cement}

In this study, conventional GIC for cementation, Ketac Cem Easymix (3M ESPE, Seefeld, Germany) was used. For the Control group (GIC), the cements were manipulated in accordance with the manufacturer's instructions, and for the Experimental group (GICEX), they were incorporated into the liquid (tartaric acid), the 18\% CLX solution during their manipulation in a proportion of one drop of tartaric acid to one drop of CLX solution, using the same dosing dropper, and was afterward spatulated with the cement powder until a solid material was obtained. ${ }^{1}$

A simple tube was soldered to the bands, Roth prescription, slot 0,022" (Dental Morelli) centralized on the vestibular surface. Afterward, they were adapted to the teeth with the aid of a band pusher, instrument for seating the band, and cemented adjacent to the gingival ridge, without aggression or ischemia of the biological space ( $1 \mathrm{~mm}$ below the marginal crest). In the groups, cementation was performed on the permanent mandibular second molars, by randomization.

The patients were evaluated and the samples collected in three time intervals: immediately before cementation (T0), 3 months after cementation (T3), and 6 months after cementation (T6).

\section{Microbiological Analysis}

For collecting crevicular fluid/dental biofilm samples, the patients were instructed not to ingest food and not to brush their teeth for a minimum period of 2 hours before collection, at time intervals $\mathrm{T0}, \mathrm{T} 3$, and $\mathrm{T} 6$.

The crevicular fluid samples were collected with the aid of sterile absorbent paper cones, caliber 20 (Dentsply, Petrópolis, Rio de Janeiro, Brazil), obtained from the mandibular second molars on the following surfaces: cervical-lingual, cervicalvestibular, mesial-interproximal, and distal-interproximal; paper cones were inserted into the gingival sulcus and held 
there for 1 minute. Paper cones were also used for collecting supragingival biofilm from the vestibular and lingual areas.

After collection, the samples were immediately transferred to a disposable plastic $1.5 \mathrm{~mL}$ Eppendorf microcentrífugal tube (Axygen, Union City, California, United States) previously weighed in a precision electronic balance (Model BG200, Gehaka, São Paulo, Brazil), stored at a temperature of $0^{\circ} \mathrm{C}$ and afterward, transported to the Microbiology and Immunology Laboratory of the Institute of Biological Sciences of the Federal University of Juiz de Fora (ICB/UFJF). After weighing the samples collected, $1 \mathrm{~mL}$ buffered saline solution was added, and each patient group was identified.

The quantity of biofilm collected was diluted and homogenized by agitation in a vortex at 12 rotations per minute, for 10 minutes, to obtain dispersion of the largest possible quantity of microorganisms. After this, the tubes were introduced into a microbiological chamber, in which serial dilutions $\left(10^{-1}-10^{-5}\right)$ were made, using $1 \mathrm{~mL}$ saline solution composed of $0.85 \%$ sodium chloride and $1 \%$ sodium thioglycolate for each $1 \mathrm{mg}$ of biofilm collected. Aliquots of $0.1 \mathrm{~mL}$ of each dilution were seeded in duplicate, with the aid of a Drigalski loop, in Petri dishes containing brain heart infusion culture medium. The materials were incubated under microaerophilic conditions ( $5 \%$ of $\mathrm{Co}^{2}$ ) by the candle flame system, at $37^{\circ} \mathrm{C}$, for 48 hours. ${ }^{19}$

The plates selected showed macroscopically visible colonies for performing the colony count readouts, which were performed by a single, previously trained and calibrated examiner (M.M.A.A.) (Kappa: 0.95).

\section{Counts and Quantification of Microorganisms}

Counting the number of CFUs was performed for the dilution that showed the growth of 30 to 300 macroscopically visible colonies.

The number of CFUs per milliliter was determined by multiplying the mean of the number of colonies counted by the factor of correction and by the inverse of the dilution factor corresponding to the dilution used in counting of the total number of microorganisms. In view of the great diversity of microorganisms present in the oral cavity, in this study, we opted for analysis of the total count of microorganisms by means of a nonselective culture.

\section{Salivary pH Evaluation}

The salivary $\mathrm{pH}$ was measured immediately before all the microbiological collections were made. The patients were instructed not to ingest any food and not to brush their teeth 2 hours before each collection. The unstimulated saliva sample was placed in a sterile plastic receptacle until $5 \mathrm{~mL}$ was obtained, and measured by means of a pH 1 to 14 indicator strip (Kasvi, Paraná, Brazil) at rest in the saliva for 10 minutes, using the colorimetric method by means of a scale for evaluating the results. The readout was taken by a single operator (J.L.S.A.) and under the same lighting condition.

\section{Color Stability Analysis}

The color stability of the teeth was evaluated by means of a visual scale using the Vitapan Classical Scale (VITA Zahnfabrik, Bad Säckingen, Germany) before placement of the banding and after its removal and cleaning of all the remnant cement. ${ }^{20}$ Scores from 1 to 16 were attributed to the colors of the scale ${ }^{21}$ ( - Fig. 2).

\section{Adhesive Remnant Index}

The bands were removed with the aid of a conical bur with a pyramid-shaped tip No. 2200 (KG Sorensen, São Paulo, Brazil) on the mesiovestibular and distolingual surfaces, without attaining the cement, and evaluated by attributing the following scores to the quantity of remnant cement: $0-$ no remnant cement on the tooth surface; 1 -less than half the tooth surface under the band covered with cement; 2-more than half the tooth surface under the band covered with cement; and 3-the entire tooth surface under the band covered with cement. $^{10}$

\section{Fixation of Bands and Gingival Evaluation}

Fixation of all the bands was evaluated through an explorer probe, with failure of fixation being considered the mobility or loosening of the band. Successful fixation was considered absence of band mobility on clinical evaluation. A macroscopic evaluation of the gingival tissues around the orthodontic bands was also performed for identifying hyperplasia or gingival inflammation at the band-gingival junction, from the measurement of the relationship between gingival insertion and cervico-occlusal height of the band using a millimeter probe. The evaluations were performed in all the time intervals, on the surfaces: cervical-lingual, cervical-vestibular, mesial-interproximal, and distal-interproximal.

\section{Statistical Analysis}

Distribution of the data was analyzed by the KolmogorovSmirnov's test (GraphPad Prism 5.0, San Diego, California, United States). For evaluation of the data between the experimental time intervals for the same cement, the nonparametric Friedman's test was used, followed by the Dunn's post hoc test for multiple comparisons $(p<0.05)$. For evaluating nonparametric data between cements in the same time interval, the nonpaired design and Mann-Whitney's tests ( $p$ $<0.05$ ) were used. Salivary $\mathrm{pH}$ was evaluated by the one-way analysis of variance and Tukey's multiple comparison tests. As regards color stability of the tooth enamel before placement and after removal of the bands, the paired $t$-test was used. The ARI was evaluated by the paired and nonpaired $t$-test $(p<0.05)$.

\begin{tabular}{|l|l|l|l|l|l|l|l|l|l|l|l|l|l|l|l|l|}
\hline Vita Shade Guide & B1 & A1 & B2 & D2 & A2 & C1 & C2 & D4 & A3 & D3 & B3 & A3.5 & B4 & C3 & A4 & C4 \\
\hline Scores & 1 & 2 & 3 & 4 & 5 & 6 & 7 & 8 & 9 & 10 & 11 & 12 & 13 & 14 & 15 & 16 \\
\hline
\end{tabular}

Fig. 2 Scores for colors according to sequence of colors on Vitapan Classical Color Guide (of lighter and darker colors). 


\section{Results}

\section{Microbiological Analysis}

The quantity of CFU demonstrated was higher and similar among the groups in the initial time interval T0 $(p=0.696)$. In the time interval of 3 months, T3, there was evidence of significant reduction in the quantity of CFU by the antimicrobial action that occurred in GICEX group in comparison with the Control ( $p=0.041)$. After the time interval of 6 months, T6, the quantity of CFU demonstrated was similar among the groups and significantly different to control $(p=0.045)$ (-Table 1).

In the comparison between the experimental time intervals, the quantity of CFU shown in GICEX group was larger in T0 when compared with the other experimental time intervals. Significant antimicrobial actions was shown in the subsequent time intervals, with statistical difference between T0 and time intervals $\mathrm{T} 3$ and T6 $(p=0.006)$. The Control group showed a similar quantity of CFU between the experimental time intervals, without statistical difference $(p=0.066)($-Table $\mathbf{1})$.

\section{Salivary pH Evaluation}

Oral pH demonstrated an increasing value from T0 to T6; statistically significant difference occurred only between experimental time intervals T0 and T6 $(p=0.022)(-$ Table 2$)$.

Table 1 Results of microbiological analyses of the cement in different experimental time intervals, in mean and standard deviation values

\begin{tabular}{|l|l|l|l|}
\hline Time & GICEX & GIC & $p$-Value \\
\hline Initial (T0) & $6.11(0.15)^{\mathrm{A}}$ & $6.05(0.24)^{\mathrm{B}}$ & 0.696 \\
\hline $3 \mathrm{mo}(\mathrm{T} 3)$ & $4.93(0.25)^{\mathrm{B}, \mathrm{a}}$ & $5.59(0.16)^{\mathrm{B}, \mathrm{b}}$ & 0.041 \\
\hline 6 mo $(\mathrm{T} 6)^{\mathrm{B}}$ & $5.28(0.23)^{\mathrm{B}, \mathrm{a}}$ & $5.90(0.09)^{\mathrm{B}, \mathrm{b}}$ & 0.045 \\
\hline -Value $^{\mathrm{b}}$ & 0.006 & 0.066 & - \\
\hline
\end{tabular}

Notes: Different superscript capital letters $\left.{ }^{(A, B}\right)$ expressed statistically significant differences in columns. Different superscript lower case letters $(\mathrm{a}, \mathrm{b})$ expressed statistically significant differences in the lines.

aFor evaluating nonparametric data between cements in the same time interval, the nonpaired design and Mann-Whitney's tests $(p<0.05)$ were used.

bFor evaluation of the data between the experimental time intervals for the same cement, the nonparametric Friedman's test was used, followed by the Dunn's post hoc test for multiple comparisons $(p<0.05)$.

Table 2 Evaluation of the influence of time on oral $\mathrm{pH}$

\begin{tabular}{|l|l|l|}
\hline \multirow{2}{*}{ Time } & pH \\
\cline { 2 - 3 } & Mean & $\begin{array}{l}\text { Standard } \\
\text { deviation }\end{array}$ \\
\hline Initial (T0) & $6.35^{\mathrm{A}}$ & 0.33 \\
\hline $3 \mathrm{mo}(\mathrm{T} 3)$ & $6.75^{\mathrm{A}, \mathrm{B}}$ & 0.63 \\
\hline $6 \mathrm{mo}(\mathrm{T} 6)$ & $7.0^{\mathrm{B}}$ & 0.47 \\
\hline$p$-Value & \multicolumn{2}{|c|}{0.022} \\
\hline
\end{tabular}

Note: $p$-Value: One-way analysis of variance followed by the Tukey's multiple comparison test $(p<0.05)$. Measurements followed by different superscript letters $\left({ }^{A, B}\right)$ expressed statistically significant difference.

\section{Color Stability Analysis}

In the tooth enamel color evaluation, no significant variations were shown between the Experimental group (GICEX) and Control for the same time interval $(p=1.000)$ and between the different time intervals for the same cement $(p=0.366)$

(-Table 3).

\section{ARI}

On a larger number of teeth, more than half the remnant cement on enamel was found (score 2 ) for both groups evaluated after 6 months, without statistically significant difference between them $(p=0.343)$ ( - Table 4$)$.

\section{Fixation of Bands and Gingival Evaluation}

For fixation of the bands, no mobility or loosening of the bands was verified during the experimental time intervals. In the gingival evaluation, no changes in gingival tissue were observed around the bands cemented in both groups GIC and GICEX, throughout the experimental time intervals.

\section{Discussion}

No previous study has verified the influence of orthodontic GIC modified by $18 \%$ CLX on the in vivo oral microbiota, salivary $\mathrm{pH}$, color of tooth enamel, and ARI. Studies conducted in vitro do not have some of the fundamental properties that modulate intraoral microbial colonization and plaque retention in patients; therefore, in vivo clinical trials must be considered to obtain clinical evidence with quality. ${ }^{19,22}$ To guarantee that this study was blind, the samples were placed in Petri dishes, and the system of identification was unknown to the examiner. ${ }^{19,23}$

Table 3 Scores of influence of GIC on color of tooth enamel for the groups, based on Vita Color scale

\begin{tabular}{|l|l|l|l|}
\hline \multirow{2}{*}{ Groups } & \multicolumn{2}{|l|}{ Times } & \multirow{2}{*}{-Value ${ }^{\mathrm{a}}$} \\
\cline { 2 - 3 } & Initial (T0) & 6 mo (T6) & \\
\hline GICEX & $6.7(3.16)$ & $7.9(4.38)$ & 0.366 \\
\hline GIC & $6.7(3.16)$ & $7.9(4.38)$ & 0.366 \\
\hline$p$-Value $^{\mathrm{b}}$ & 1.000 & 1.000 & \\
\hline
\end{tabular}

Abbreviation: GIC, glass ionomer cement.

${ }^{a}$ For evaluation of parametric data for each cement in different time intervals, the paired t-test was used $(p<0.05)$.

bFor evaluation of parametric data for each cement in the same time interval, the nonpaired $t$-test was used $(p<0.05)$.

Table 4 ARI scores ${ }^{\mathrm{a}}$ and mean values are presented in the Groups

\begin{tabular}{|l|l|l|l|l|l|}
\hline \multirow{2}{*}{ Groups } & \multicolumn{4}{|l|}{ ARI score } & \multirow{2}{*}{ Mean } \\
\cline { 2 - 5 } & $\mathbf{0}$ & $\mathbf{1}$ & $\mathbf{2}$ & $\mathbf{3}$ & \\
\hline GICEX & 0 & 2 & 7 & 1 & 1.9 \\
\hline GIC & 0 & 0 & 9 & 1 & 2.1 \\
\hline$p$ Value $^{\mathrm{b}}$ & - & - & - & - & 0.343 \\
\hline
\end{tabular}

Abbreviations: ARI, adhesive remnant index; GIC, glass ionomer cement. ${ }^{a} 0$, no remnant cement; 1 , less than half the remnant cement; 2 , more than half of the remnant cement; 3 , all the remnant cement.

bParametric data, nonpaired $t$-test $(p<0.05)$. 
In the microbiological, ${ }^{19,23}$ the present study demonstrated significant reduction in the total CFU count of GICEX group in the time interval of 3 months (T3) when compared with Control group (GIC). In the time interval of 6 month (T6), the quantities of CFU remained statistically similar to those of $\mathrm{T} 3$, in relation to control. These findings were in agreement with those of other studies that demonstrated the antimicrobial action of GIC modified by CLX, against S. mutans, by means of gradual release of CLX ${ }^{16,24}$ for $65^{1}$ and 90 days ${ }^{16}$ in in vitro analyses. Due to the porous mesh of conventional GIC and the substantivity ${ }^{25,26}$ of CLX, it has been suggested that a renewed antimicrobial effect ${ }^{9,16}$ could effectively be expected throughout the course of orthodontic treatment, resulting from the superficial erosion, exposing a new GIC surface for the release of CLX. ${ }^{27}$ This would allow the CLX to react with cell structures and lead to direct damage to or inhibition of bacterial cell metabolism.

Studies ${ }^{5,6}$ have demonstrated that the diversity of devices used in orthodontic treatment favored a higher concentration of microorganisms, thereby increasing the microbial flora, and consequently, a leading to a reduction in salivary $\mathrm{pH}$, and the development of the main oral diseases such as caries and periodontal diseases. ${ }^{19,28}$ The present study demonstrated a gradual increase in the salivary $\mathrm{pH}$ from the time interval T0 to T6, with significant differences between the time intervals. The $\mathrm{pH}$ value ranged from 6.35 in the beginning to 7.0 on conclusion of the study, an increase below the value of variation from normality in adults (6.2-7.4) ${ }^{28-30}$ Variations in $\mathrm{pH}$ due to dietary products or the conversion of sugar into acid by dental biofilm determine the limit of the capacity of saliva for protecting the teeth against caries and periodontal disease, with $\mathrm{pH} 5.5$ being the critical level. ${ }^{31,32}$ In this study, it could be verified that during the entire experimental time interval, the patients had $\mathrm{pH}$ values above the level considered critical; however, the composition and quantity of salivary secretions may also vary with age and influence bacterial adherence. ${ }^{19,33,34}$ It is suggested that oral $\mathrm{pH}$ was not a determinant factor in the quantity of microorganisms but may have been an influencing factor at some time during orthodontic treatment.

With regard to evaluating the color of tooth enamel, the Vita Color guide is validated and has been used in different studies. ${ }^{21,35}$ Visual evaluation guided by the color scale depends on some variables, ${ }^{36,37}$ such as the light source, characteristics of the tooth, training, and experience of the observer. ${ }^{38}$ These variables were considered and standardized by the observer in this study, Kappa (0.90). The change in color of tooth enamel resulting from oral mouth washes, such as CLX has been reported in the literature ${ }^{39,40}$ as being an effect of the high substantivity of the active principle combined with prolonged use of the mouth wash. However, the results of the present study demonstrated that the CLX incorporated into the GIC was not capable of promoting change in color of the tooth enamel, even in the long term.

Studies have demonstrated that the incorporation of $18 \%$ CLX into the orthodontic GIC did not significantly influence the diametral tensile strength, resistance to compression, shear bond strength, or the microhardness ${ }^{1,10}$ of the GICs, ${ }^{16}$ in vitro. In this study, the ARI showed no significant difference between the conventional CIC and that modified by CLX, and the bands of all the patients showed no mobility and failure to survive throughout the experimental period.

These results corroborate with research ${ }^{1,10,16}$ that demonstrated that the inclusion of CLX in the ionomeric cement did not impair clinical performance when considering disturbances such as fracture, solubilization, infiltration, and consequently, decalcification of the dental enamel, and periodontal disease adjacent to the orthodontic bands. ${ }^{1,10}$ The ARI demonstrated that more than half or all of the remaining adhesives remained on the dental surface after removing the band, regardless of the addition of CLX, demonstrating that CLX did not interfere with cement adhesion to dental enamel. ${ }^{16}$

Different studies ${ }^{10,41,42}$ in which conventional GIC was used for cementing bands to the first molar have revealed failure rates ranging between 0 and 26\% in different periods of follow-up and mechanics applied to the bands, which makes comparisons difficult. However, the authors have reported a failure rate of $18 \%$ was expected in bands submitted to the mechanics of force of extraoral appliances connected to these ${ }^{43}$ irrespective of the type of cement used. This corroborated the findings of the present study, in which all the patients were treated with the Straight Wire appliance, and accessories such as the extraoral type were not used on the bands, resulting in demonstration of a failure rate of $0 \%$.

The present study performed randomization of the patients, and the side on which cementation of the bands occurred/groups in the mandibular arch, based on studies that reported that the age ${ }^{41,43}$ and gender, ${ }^{41,43}$ and localization $^{41,44}$ of the band in the arch were not considered significant factors that affected the rates of failure of the band.

Previous studies about the tissue biocompatibility of GIC modified by CLX ${ }^{9,16}$ have demonstrated a low level of tissue cytotoxicity, without significant difference between concentrations of 10 and $18 \%$ CLX after 30 days of contact of GIC with subcutaneous tissue of rats. However, the cytotoxicity of CLX at higher concentrations has been reported, ${ }^{25,45}$ but when it is associated with GICs, this potential may be retarded by the slow release of CLX into the crystallized network of the cement. ${ }^{16}$ In this study, clinically no gingival changes were observed in the tissues around the bands cemented with GIC modified by CLX, over the experimental time intervals, which suggests tissue biocompatibility with cement. New randomized clinical trials with longer times of evaluation may trace a more long-term behavior of these modified cements. In general, the addition of CLX to the cements was shown to be a highly promising method for obtaining an antibacterial GIC for orthodontic cementation, which associated with the fluoride in tooth pastes, mouth washes, and/or varnishes with cariostatic action ${ }^{46}$ could provide the oral medium with stronger and greater protection.

\section{Conclusion}

- The incorporation of $18 \%$ CLX into GIC, in the 6-month period: 
- Demonstrated effective antibacterial action

- Allowed good bonding of the cement to enamel, based on the ARI

- Demonstrated a high rate of survival of the bonded bands

- Did not change the color of tooth enamel

- Maintained the salivary pH at physiological levels.

\section{Funding}

This study was financed in part by the Coordenação de Aperfeiçoamento de Pessoal de Nível Superior, Brazil (CAPES) - Finance Code 001.

\section{Conflict of Interest}

None declared.

\section{References}

1 FarretMM, de LimaEM, MotaEG, Oshima HM, Barth V, de Oliveira SD. Can we add chlorhexidine into glass ionomer cements for band cementation? Angle Orthod 2011;81(3):496-502

2 Almeida Mesquita J, Lacerda-Santos R, Pina Godoy G, Franscisco Weege Nonaka C, Muniz Alves P. Morphological and immunohistochemical analysis of the biocompatibility of resin-modified cements. Microsc Res Tech 2017;80(5):504-510

3 Mesquita JA, Lacerda-Santos R, Sampaio GAM, Godoy GP, Nonaka CFW, Alves PM. Evaluation in vivo of biocompatibility of differents resin-modified cements for bonding orthodontic bands. An Acad Bras Cienc 2017;89(3, Suppl):2433-2443

4 Vandersluis YR, Suri S. Infective endocarditis and orthodontic implications in children: a review of the literature. Am J Orthod Dentofacial Orthop 2020;157(1):19-28

5 Bergamo AZ, Nelson-Filho P, Romano FL, et al. Gingival crevicular fluid volume and periodontal parameters alterations after use of conventional and self-ligating brackets. J Orthod 2016;43(4):260-267

6 Reichardt E, Geraci J, Sachse S, et al. Qualitative and quantitative changes in the oral bacterial flora occur shortly after implementation of fixed orthodontic appliances. Am J Orthod Dentofacial Orthop 2019;156(6):735-744

7 Pandis N, Vlachopoulos K, Polychronopoulou A, Madianos P, Eliades T. Periodontal condition of the mandibular anterior dentition in patients with conventional and self-ligating brackets. Orthod Craniofac Res 2008;11(4):211-215

8 Aguilar-Perez D, Vargas-Coronado R, Cervantes-Uc JM, et al. Antibacterial activity of a glass ionomer cement doped with copper nanoparticles. Dent Mater J 2020;39(3):389-396

9 Lacerda-Santos R, Sampaio GA, Moura MdeF, et al. Effect of different concentrations of chlorhexidine in glass-ionomer cements on in vivo biocompatibility. J Adhes Dent 2016;18(4): 325-330

10 Millett DT, Doubleday B, Alatsaris M, et al. Chlorhexidinemodified glass ionomer for band cementation? An in vitro study. J Orthod 2005;32(1):36-42

11 Ripari F, Cera A, Freda M, Zumbo G, Zara F, Vozza I. Tea tree oil versus chlorhexidine mouthwash in treatment of gingivitis: a pilot randomized, double blinded clinical trial. Eur J Dent 2020;14(1):55-62

12 Torres-Rosas R, Torres-Gómez N, Moreno-Rodríguez A, García-Contreras R, Argueta-Figueroa L. Anti-inflammatory and antibacterial activity of the chitosan/chlorhexidine gel commercial preparation for postexodontia treatment: an in vitro study. Eur J Dent 2020;14(3):397-403

13 Coelho AS, Laranjo M, Gonçalves AC, et al. Cytotoxic effects of a chlorhexidine mouthwash and of an enzymatic mouthwash on human gingival fibroblasts. Odontology 2020;108(2):260-270
14 Meneses IHC, Sampaio GAM, Carvalho FG, et al. In vivo biocompatibility, mechanical, and antibacterial properties of cements modified with propolis in different concentrations. Eur J Dent 2020;14(1):77-84

15 Sampaio GAM, Lacerda-Santos R, Cavalcanti YW, Vieira GHA, Nonaka CFW, Alves PM. Biocompatibility of ionomeric cements modified by red propolis: a morphological and immunohistochemical analysis. J Adhes Dent 2020;22(5):515-522

16 Sampaio GM, de Meneses IH, de Carvalho FG, et al. Antimicrobial, mechanical and biocompatibility analysis of chlorhexidine digluconate-modified cements. J Clin Exp Dent 2020;12(2):e178-e186

17 Wolfe DA, Nonparametrics: Statistical Methods Based on Ranks and Its Impact on the Field of Nonparametric Statistics. In: Rojo J. (eds) Selected Works of E.L. Lehmann. Selected Works in Probability and Statistics. Springer, Boston, MA;2012: $1101-1110$

18 DanielsC,RichmondS.The development of theindex of complexity, outcome and need (ICON). J Orthod 2000;27(2):149-162

19 do Nascimento LE, Pithon MM, dos Santos RL, et al. Colonization ofStreptococcus mutanson esthetic brackets: self-ligating vs conventional. Am J Orthod Dentofacial Orthop 2013; 143(4, Suppl):S72-S77

20 Brandt J, Nelson S, Lauer HC. von Hehn U, Brandt S. In vivo study for tooth colour determination-visual versus digital. Clin Oral Investig 2017;21(9):2863-2871

21 Meireles SS, Demarco FF, dos Santos IdaS, Dumith SdeC, Bona AD. Validation and reliability of visual assessment with a shade guide for tooth-color classification. Oper Dent 2008;33(2): 121-126

22 Jadad AR, Moore RA, Carroll D, et al. Assessing the quality of reports of randomized clinical trials: is blinding necessary? Control Clin Trials 1996;17(1):1-12

23 Brusca MI, Chara O, Sterin-Borda L, Rosa AC. Influence of different orthodontic brackets on adherence of microorganisms in vitro. Angle Orthod 2007;77(2):331-336

24 Yadiki JV, Jampanapalli SR, Konda S, Inguva HC, Chimata VK. Comparative evaluation of the antimicrobial properties of glass ionomer cements with and without chlorhexidine gluconate. Int J Clin Pediatr Dent 2016;9(2):99-103

25 Lessa FC, Aranha AM, Nogueira I, Giro EM, Hebling J, Costa CA. Toxicity of chlorhexidine on odontoblast-like cells. J Appl Oral Sci 2010;18(1):50-58

26 Rölla G, Melsen B. On the mechanism of the plaque inhibition by chlorhexidine. J Dent Res 1975;54 Spec No B:B57-B62

27 Hoszek A, Ericson D. In vitro fluoride release and the antibacterial effect of glass ionomers containing chlorhexidine gluconate. Oper Dent 2008;33(6):696-701

28 Dos Santos AA, Pithon MM, Carlo FG, et al. Effect of time and $\mathrm{pH}$ on physical-chemical properties of orthodontic brackets and wires. Angle Orthod 2015;85(2):298-304

29 Aframian DJ, Davidowitz T, Benoliel R. The distribution of oral mucosal $\mathrm{pH}$ values in healthy saliva secretors. Oral Dis 2006;12(4):420-423

30 Sifakakis I, Papaioannou W, Papadimitriou A, Kloukos D, Papageorgiou SN, Eliades T. Salivary levels of cariogenic bacterial species during orthodontic treatment with thermoplastic aligners or fixed appliances: a prospective cohort study. Prog Orthod 2018;19(1):25

31 Serratine ACP, Silva MRM. Validation of a simplified method for evaluation of salivary $\mathrm{pH}$ in children. Pesqui Bras Odontopediatria Clin Integr 2009;9(2):217-221

32 Dawes $\mathrm{C}$. What is the critical $\mathrm{pH}$ and why does a tooth dissolve in acid? J Can Dent Assoc 2003;69(11):722-724

33 Ramberg P, Axelsson P, Lindhe J. Plaque formation at healthy and inflamed gingival sites in young individuals. J Clin Periodontol 1995;22(1):85-88 
34 Ristic M, Vlahovic Svabic M, Sasic M, Zelic O. Clinical and microbiological effects of fixed orthodontic appliances on periodontal tissues in adolescents. Orthod Craniofac Res 2007;10(4):187-195

35 Paul S, Peter A, Pietrobon N, Hämmerle $\mathrm{CH}$. Visual and spectrophotometric shade analysis of human teeth. J Dent Res 2002;81(8):578-582

36 Watts A, Addy M. Tooth discolouration and staining: a review of the literature. Br Dent J 2001;190(6):309-316

37 Hassel AJ, Koke U, Schmitter M, Beck J, Rammelsberg P. Clinical effect of different shade guide systems on the tooth shades of ceramic-veneered restorations. Int J Prosthodont 2005;18(5):422-426

38 Joiner A. The bleaching of teeth: a review of the literature. J Dent 2006;34(7):412-419

39 Bagis B, Baltacioglu E, Özcan M, Ustaomer S. Evaluation of chlorhexidine gluconate mouthrinse-induced staining using a digital colorimeter: an in vivo study. Quintessence Int 2011; 42(3):213-223

40 Zanatta FB, Antoniazzi RP, Rösing CK. Staining and calculus formation after $0.12 \%$ chlorhexidine rinses in plaque-free and plaque covered surfaces: a randomized trial. J Appl Oral Sci 2010;18(5):515-521
41 Hodges SJ, Gilthorpe MS, Hunt NP. The effect of micro-etching on the retention of orthodontic molar bands: a clinical trial. Eur J Orthod 2001;23(1):91-97

42 Millett DT, McCabe JF, Bennett TG, Carter NE, Gordon PH. The effect of sandblasting on the retention of first molar orthodontic bands cemented with glass ionomer cement. Br J Orthod 1995;22(2):161-169

43 Millett DT, Hallgren A, McCluskey LA, et al. A clinical retrospective evaluation of 2 orthodontic band cements. Angle Orthod 2001;71(6):470-476

44 Stirrups DR. A comparative clinical trial of a glass ionomer and a zinc phosphate cement for securing orthodontic bands. Br J Orthod 1991;18(1):15-20

45 Walsh T, Oliveira-Neto JM, Moore D. Chlorhexidine treatment for the prevention of dental caries in children and adolescents. Cochrane Database Syst Rev 2015;(4):CD008457

46 Jenatschke F, Elsenberger E, Welte HD, Schlagenhauf U. Influence of repeated chlorhexidine varnish applications on mutans streptococci counts and caries increment in patients treated with fixed orthodontic appliances. J Orofac Orthop 2001;62(1):36-45 